\title{
The Force is With You - The Apparent Vertical Filter Concept
}

\author{
Carsten Seehof ${ }^{1}$, Jan-Philipp Buch ${ }^{2}$ and Christian Raab ${ }^{3}$ \\ German Aerospace Center (DLR), Institute of Flight Systems, Braunschweig, Germany
}

\begin{abstract}
Supplying aircraft pilots with adequate motion cues in flight simulators is still an important issue. The main problem is the limited space envelope of motion systems that makes an ideal replication of accelerations impossible. As a consequence those translational acceleration signals cannot be provided directly and need to be transformed to angular attitudes or scaled down depending on the time in which they appear. State-of-the-art motion drive algorithms like the classic washout filter algorithm are using sets of high- and low-pass filters in combination with gain factors to achieve such a response. But those control methods do not distinguish between the causes of accelerations with respect to flight maneuvers. The Apparent Vertical Filter developed by the German Aerospace Center provides a different approach to this problem. The current paper gives an overview of how the problem of false motion cues could be adessed within a number of idealized maneuvers. As an example the filter input data of a coordinated turn maneuver will be defined. The constraints of current filter methods using the classic washout filter will be discussed. The paper gives an overview of how the apparent vertical filter deals with the problem and how it is structured. Finally, the responses of both filter methods, the AVF anf the CWA, to aircraft model data are compared.
\end{abstract}

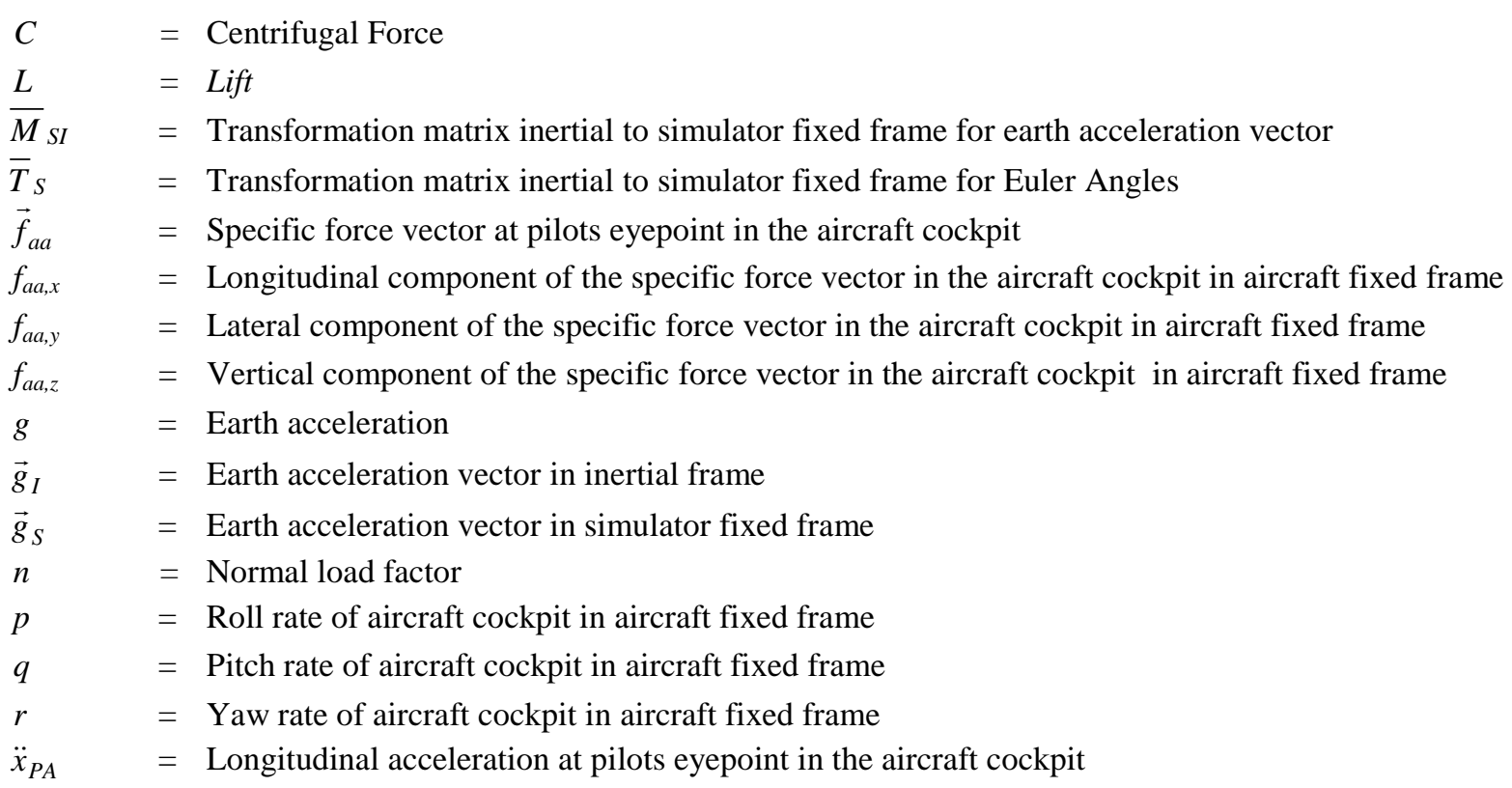

${ }^{1}$ Research Scientist, Flight Dynamics and Simulation, Lilienthalplatz 7, 38108 Braunschweig, Carsten.Seehof@dlr.de.

${ }^{2}$ Research Scientist, Flight Dynamics and Simulation, Lilienthalplatz 7, 38108 Braunschweig, Jan-Philipp.Buch@dlr.de.

${ }^{3}$ Research Scientist, Flight Dynamics and Simulation, Lilienthalplatz 7, 38108 Braunschweig, Christian Raab@dlr.de. 


$\begin{array}{ll}\vec{x}_{S} & =\text { Position vector of pilots eyepoint in the simulator cockpit } \\ \ddot{y}_{P A} & =\text { Lateral acceleration at pilots eyepoint in the aircraft cockpit } \\ \ddot{y}_{S} & =\text { Lateral acceleration at pilots eyepoint in the simulator cockpit } \\ \ddot{z}_{P A} & =\text { Vertical acceleration at pilots eyepoint in the aircraft cockpit } \\ \vec{\beta}_{S} & =\text { Attitude anglesof pilots eyepoint } \\ \phi & =\text { Aircraft roll attitude } \\ \phi_{S} & =\text { Simulator cockpit roll attitude } \\ \varphi & =\text { Lateral apparent vertical angle } \\ \varphi_{f} & =\text { Lateral apparent vertical angle due to lateral acceleration } \\ \varphi_{\omega} & =\text { Lateral apparent vertical angle due to roll attitude } \\ \theta & =\text { Aircraft pitch attitude } \\ \vec{\omega}_{a a} & =\text { Angular velocity vector in the aircraft cockpit } \\ \omega_{a a, x} & =\text { Roll rate signal of aircraft model } \\ \omega_{a a, y} & =\text { Pitch rate signal of aircraft model } \\ \omega_{a a, z} & =\text { Yaw rate signal of aircraft model }\end{array}$

\section{Introduction}

$\mathrm{T}$ The acceptance of motion simulation in flight training is an ongoing discussion. At least three lines of argument can be distinguished: First there is the economic argument: From the operator's point of view the direct and indirect costs resulting from operating a motion based full flight simulator demand an equivalent in the form of increased safety, improved training effectiveness or savings from reduced aircraft operating time. At least airlines operating small or commuter aircraft have to decide whether to perform mandatory training on an aircraft or contract simulator time [1] [2]. Secondly the quality of motion simulation is an important topic: Across academia the discussion of how to define generally accepted procedures to objectively prove the quality of motion systems and their transfer-of-training value is still open [3] [4]. The third argument comes from the users' side: pilots are still sensing interfering forces resulting from the fact that reaction forces, e. g. the centrifugal force during coordinated turns cannot be replicated due to the limited envelope space that is available for motion systems [5]. Summarizing all arguments above, it can be said that even though objective evaluation methods are to be discussed further, it is generally accepted that efforts have to be made to improve subjective motion cueing [6]. Due to economic aspects new methods for motion drive algorithms are consistently under development because those methods can be used in existing motion systems without any change to the hardware.

One example of a flight maneuver that is difficult to reproduce in flight simulators is a coordinated turn. In this paper a coordinated turn will be defined as a turn with a certain roll attitude where the centrifugal force ensures that no side force is felt by the pilot in the aircraft cockpit [Fig. 1]. As a consequence a g-load factor is sensed in the aircraft while the apparent vertical remains along the vertical axis of the cockpit assuming small pitch angles. It is obvious that the vertical load factor cannot be replicated in the simulator cabin due to the fact that it is neither possible to induce another external force, e. g. centrifugal force on the cabin except gravity, nor to accelerate the cabin in a vertical direction for the duration of the turn.

This leads to the problem that rolling the simulator cabin will induce side forces that are felt in the simulator resulting from the change in g-force orientation. Leaving the simulator cabin in a neutral position will avoid false side forces but will withhold rotational cues from the pilot. Both effects can be balanced by rotating the simulator cabin in the direction of the aircraft roll while compensating the resulting side forces by accelerating the cabin laterally. Because of limited translational space this acceleration needs to be stopped, which requires a corresponding roll-back maneuver of the cabin to prevent cabin-fixed side forces. Most current motion drive algorithms use methods to compensate side forces due to simulator roll attitudes. 


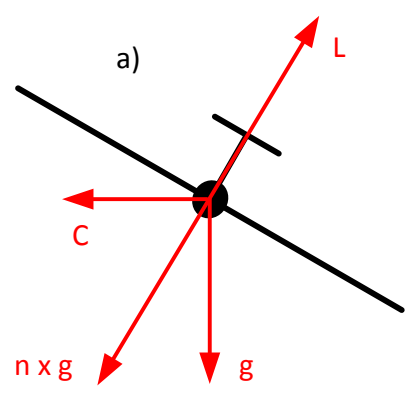

b)

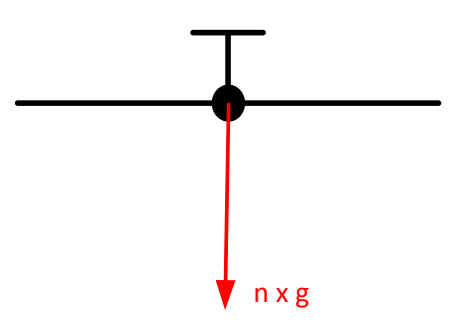

c)

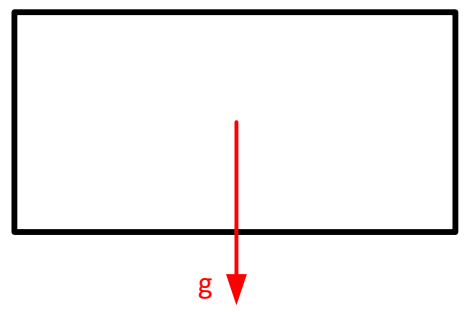

Figure 1. Forces appearing during a coordinated turn in an aircraft in the earth fixed frame (a), how they are sensed in the aircraft in the body-fixed frame (b) and what can be achieved in a simulator cabin in cabin-fixed coordinates (c)

One current approach is the Lateral Motion Maneuver ( $\left.\mathrm{LM}^{2}\right)$ filter [7] [8], which is able to compensate for some of the adverse effects of lateral maneuvers but is not valid for longitudinal accelerations that appear for example during take-off runs, climb and descent maneuvers, as well as turns during taxiing on the ground. But even the most common filter algorithm, the classical washout filter algorithm (CWA), includes a method for unwanted side force compensation that will be discussed in the following section.

The latest filter method is the Apparent Vertical Filter (AVF) developed by DLR. This filter method analyzes the acceleration signals with respect to the angular attitude of the aircraft. To demonstrate the characteristics of the AVF compared to the CWA an idealized set of input signals will be defined for a coordinated turn. The corresponding response of a CWA to this signal will be discussed. After that the AVF will be presented and its response to the same input signal will be shown. Finally both filter methods will be compared using recorded simulator data of a coordinated turn with an Airbus A320 flight test data.

\section{General Characteristics in a Coordinated Turn}

A coordinated turn within this context describes a lateral aircraft maneuver. The initial state of the aircraft is an un-accelerated, levelled and straight flight. By deflecting ailerons, rudder and, if applicable, spoilers a roll is induced in a way that no side force can be observed. When reaching a certain roll attitude the roll motion will be stopped by using all control surfaces in a way that, again, no side force will occur. The resultant of the centrifugal and gravitational forces will be compensated for by a roll attitude. By doing so the apparent vertical remains aligned to the vertical axis of the aircraft cabin during the complete maneuver.

The corresponding input signals for a turn may be idealized in a way shown by Fig. 2. The roll rate is a step input signal of $10 \%$ for 3 seconds. A roll rate of this size is typical for a passenger aircraft of Airbus A320 or Boeing B737 size. After initializing the turn, an inversed input stops the roll rate. The specific lateral force remains zero for the entire maneuver. The vertical specific force matches gravitational acceleration at the start of the maneuver. For levelled coordinated turns the g-load factor is given by a function only depending on the roll angle of the aircraft. With the roll attitude given the vertical specific force therefore is

$$
\mathrm{f}_{(\mathrm{a}, \mathrm{z})}=\frac{-\mathrm{g}}{\cos (\phi)}
$$



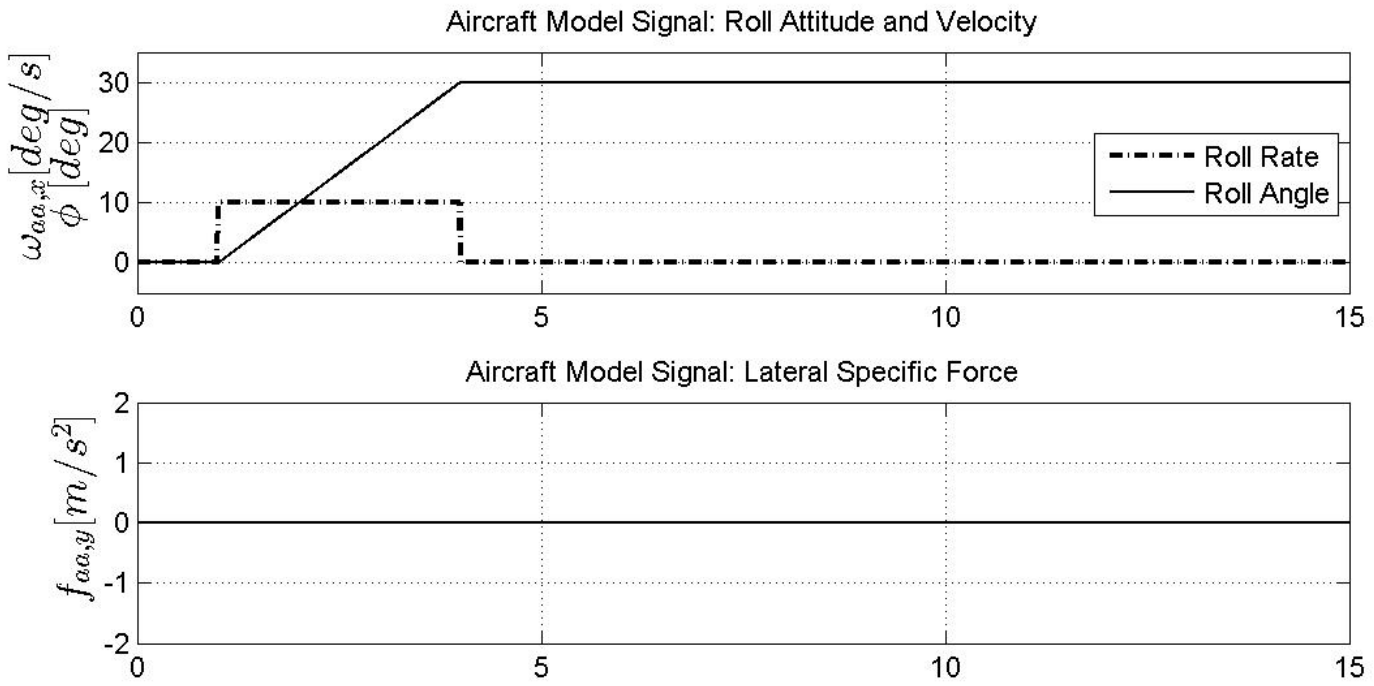

Aircraft Model Signal: Vertical Specific Force

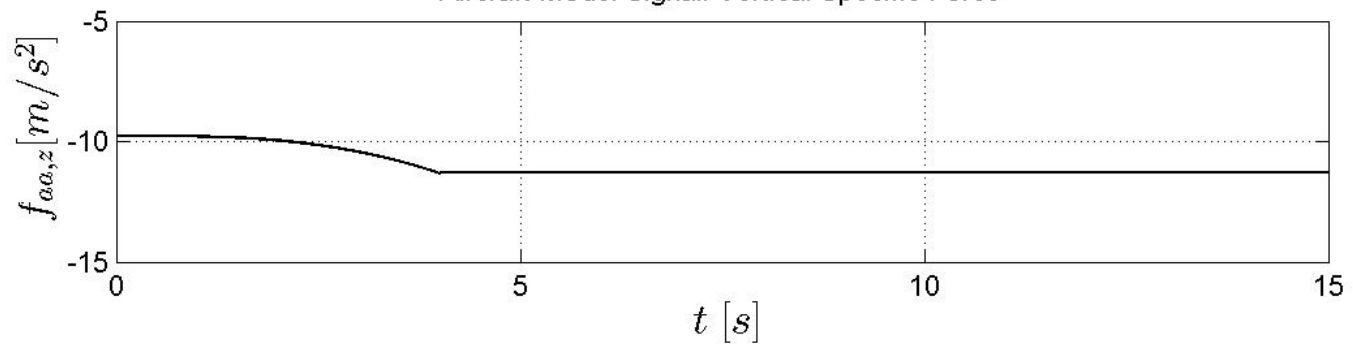

Figure 2. Idealized filter input signals for a coordinated turn

\section{A. System Response of a CWA to a Coordinated Turn}

At least since the first CWA research report was published by the University of Toronto [9], the CWA has become the most popular and probably the best analyzed motion drive algorithm in use in flight training simulators. And even though today a number of derivatives of the published CWA are in use, all of them are based upon the algorithm presented in [9]. For that reason this algorithm is used as a reference algorithm for the research presented in this paper. The response to the input signals defined above will be discussed using the following CWA structure.

The input signals of the translational and the tilt-coordination channel are the specific forces. These are defined by

$$
\overrightarrow{\mathrm{f}}_{\mathrm{aa}}=\left(\begin{array}{c}
\mathrm{f}_{\mathrm{aa}, \mathrm{x}} \\
\mathrm{f}_{\mathrm{aa}, \mathrm{y}} \\
\mathrm{f}_{\mathrm{aa}, \mathrm{z}}
\end{array}\right)=\left(\begin{array}{l}
\ddot{\mathrm{x}} \\
\ddot{\mathrm{y}} \\
\ddot{\mathrm{z}}
\end{array}\right)_{\mathrm{PA}}-\left(\begin{array}{c}
-\sin (\theta) \\
\sin (\phi) \cdot \cos (\theta) \\
\cos (\phi) \cdot \cos (\theta)
\end{array}\right) \cdot g
$$

while the rotational velocities are given by

$$
\vec{\omega}_{\mathrm{aa}}=\left(\begin{array}{c}
\mathrm{p} \\
\mathrm{q} \\
\mathrm{r}
\end{array}\right)=\left(\begin{array}{c}
\omega_{\mathrm{aa}, \mathrm{x}} \\
\omega_{\mathrm{aa}, \mathrm{y}} \\
\omega_{\mathrm{aa}, \mathrm{z}}
\end{array}\right)
$$




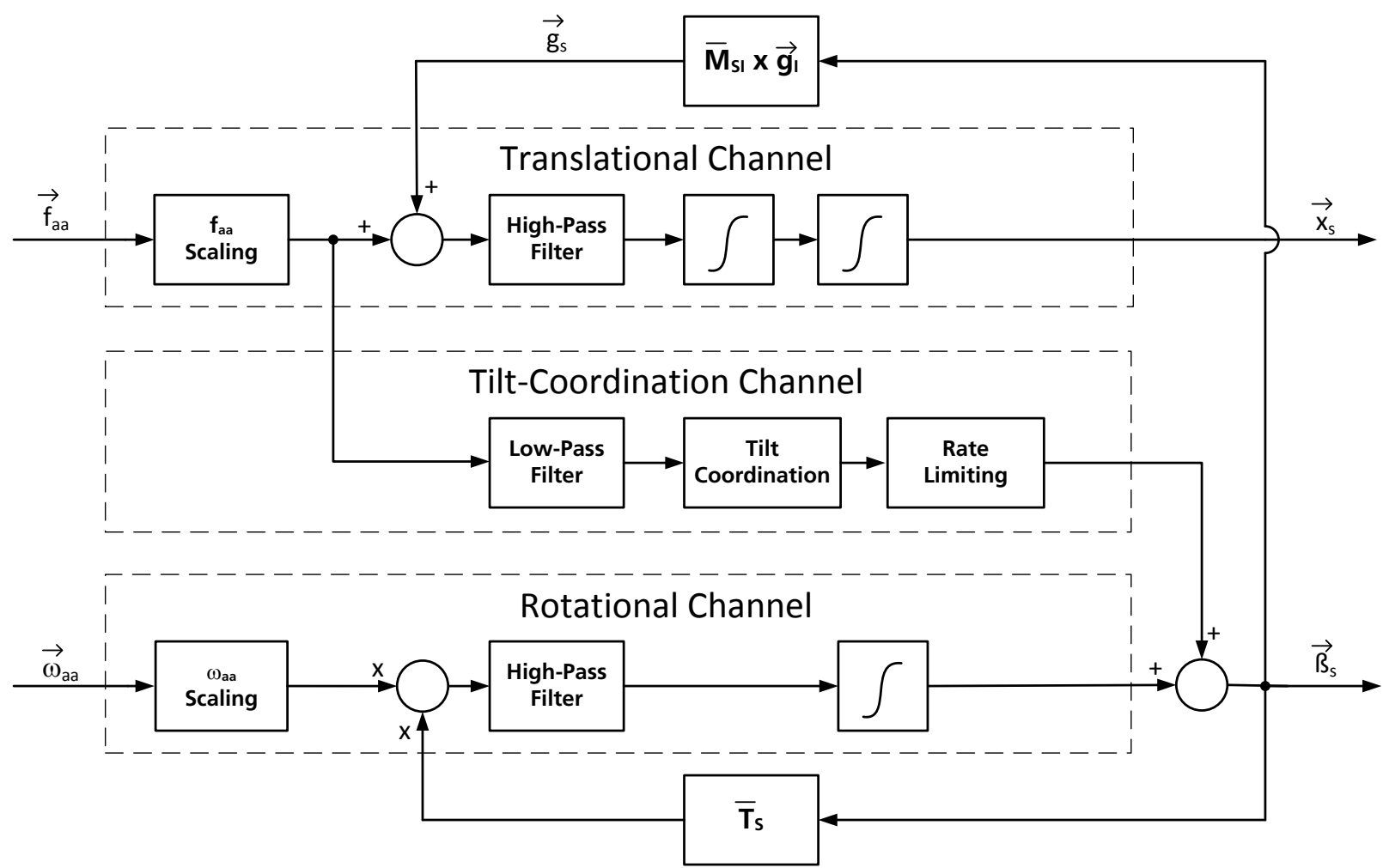

Figure 3. Classical washout filter algorithm according to [9]

The output signals are the position vector and the attitude vector of the pilot eyepoint. Due to the high-pass filter of the translational channel short term accelerations will lead to translational accelerations of the motion system, while long term signals will lead to attitude angles using low-pass filters. The short term rotational rates input signal, which is sent via the high-pass filter of the rotational channel, will lead to attitude angles too, while long term input signals will be filtered out. For the idealized coordinated turn it is assumed that longitudinal and lateral specific forces as well as the pitch attitude can be neglected. The yaw angular velocity is small with respect to the roll rate and has hardly any relevance to the lateral motion system response. The following figure shows the same CWA structure as above with all parts greyed-out that are either not relevant for lateral forces or negligible due to the input signals defined according to the preceeding assumptions

$$
\overrightarrow{\mathrm{f}}_{\mathrm{aa}}=\left(\begin{array}{c}
0 \\
0 \\
\frac{1}{\cos (\phi)}
\end{array}\right) \cdot g
$$

and

$$
\vec{\omega}_{\mathrm{aa}}=\left(\begin{array}{c}
\omega_{\mathrm{aa}, \mathrm{x}} \\
0 \\
0
\end{array}\right)
$$




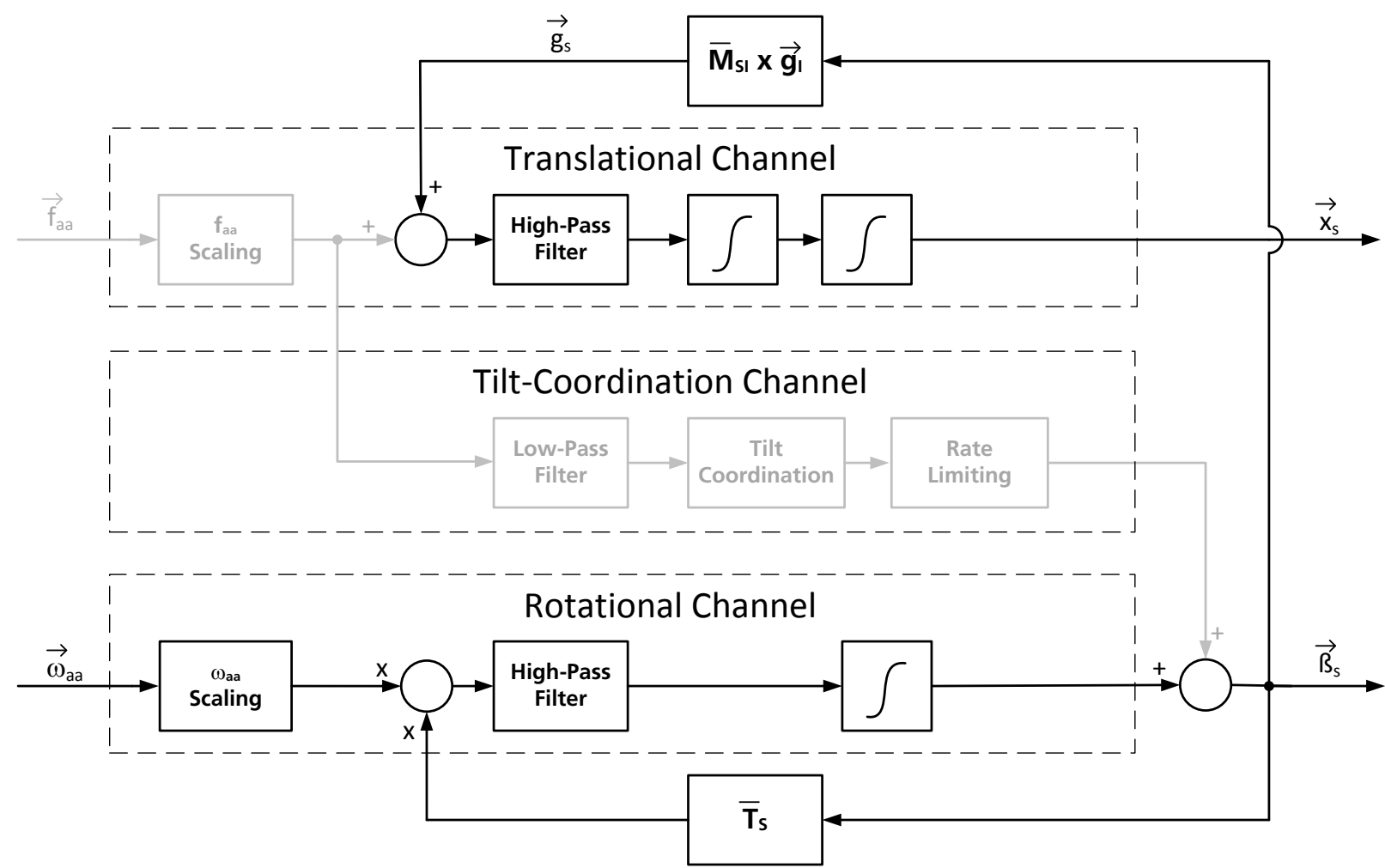

Figure 4. Classical washout filter algorithm processing an idealized coordinated turn input signal

Due to rotational input signals the simulator cabin starts rolling according to the aircraft turn. Using the resulting cabin attitude the gravity vector is transformed to the simulator fixed frame and added to the translational channel. Due to this feedback loop a compensatory lateral acceleration signal is supposed to ensure that unintended side forces resulting from cabin roll are avoided. Unfortunately this compensatory signal is high-pass filtered which impedes a perfect lateral compensation of the side force due to the cabin roll. In principle it is possible to add the transformed gravity vector after the high-pass filter is applied resulting in a well-compensated roll maneuver. But, inevitably, this leads to noticeable wrong cues whenever the specific force vector is not zero due to high frequency residues.

Fig. 5 shows the response of a classical washout filter algorithm with respect to an idealized coordinated turn. The filter tuning corresponds to a setting of a comparable filter used in an approved Boeing B737 training simulator. As expected the roll velocity input signal results in a high-pass filter response. The second graph shows the lateral specific force of the ideal maneuver and the force felt in the simulator cabin. It is easy to see that a side force will be observed by the pilot in the simulator which does not correspond to the input signal. As a consequence this side force could lead to confusion and to an incorrect response by the pilot. Therefore simulator operators usally reduce the gain of the lateral signals to a value of 0.25 to 0.5 to suppress those false cues. This results in extremely reduced roll rates. Consequently, this strategy prevents pilots from sensing an important motion cue. It will be shown below that neither this reduction nor the false sideforces inevitably have to be accepted. In fact, both can be avoided if the motion drive algorithm is able to compensate the side force. The last graph shows that any additional vertical specific force cannot be replicated by the simulator and, therefore, the vertical specific force felt in the simulator corresponds approximately to $\mathrm{g}$ for the whole duration of the maneuver. 

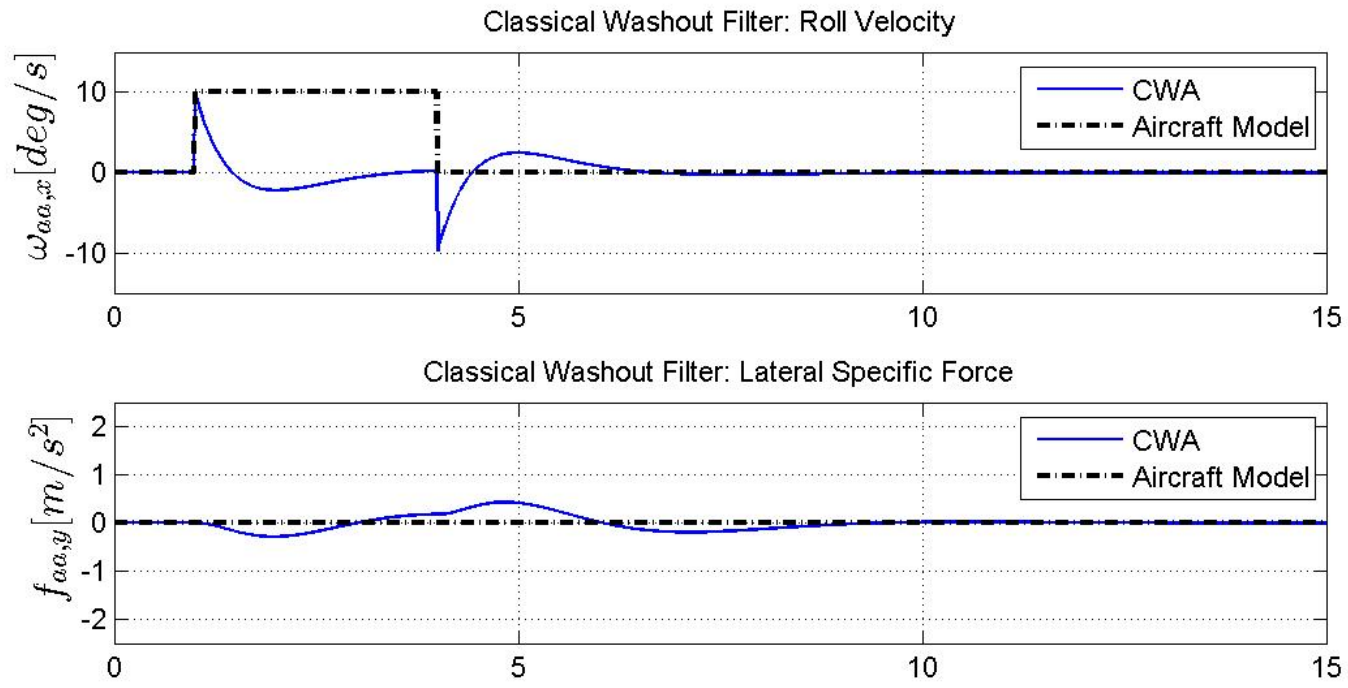

Classical Washout Filter: Vertical Specific Force

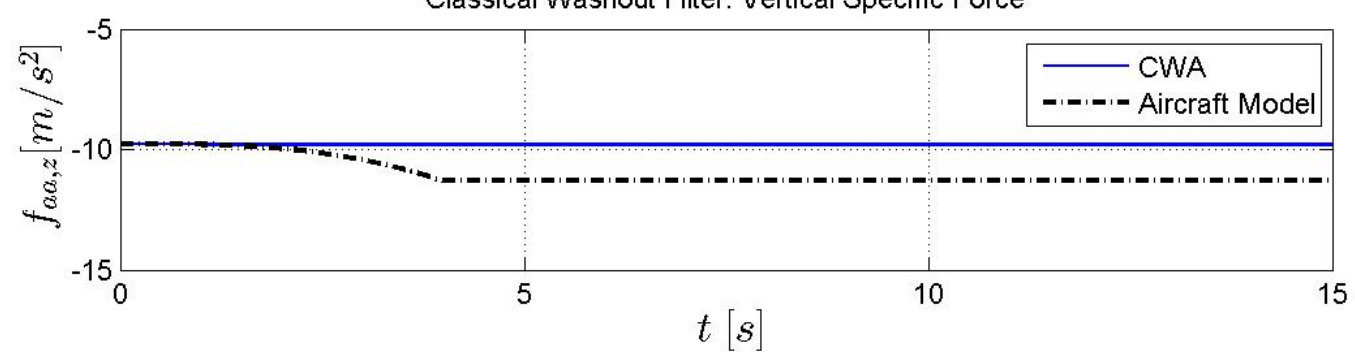

Figure 5. Classical washout filter algorithm reponse to an idealized coordinated turn

\section{B. The AVF and its Response to a Coordinated Turn}

The main idea of the Apparent Vertical Filter is to represent specific forces in the simulator with respect to the perceived apparent vertical angle rather than the correct quantity. the orientation of the force vector shall be correct as far as possible, while the length of the vector shall be adapted to the technical constraints of the motion system. This is achieved by comparing the current aircraft attitude angles with the current specific force vector cued in the cockpit. It should be noted that any translational acceleration and any change of attitude angle leads to a change of the cued apparent vertical different from normal gravity. As a consequence both specific forces and angular attitudes can be expressed as a change of the apparent vertical. Fig. 6 shows the principle of the apparent vertical filter for the processing of lateral maneuver input data.

Any lateral maneuver of an aircraft is characterized by a specific apparent vertical angle induced by a roll rate, specific side force in relation to gravity or both. The apparent vertical angle due to the aircraft angular attitude can be found by integrating the roll rate

$$
\varphi_{\omega}=\int \omega_{\mathrm{aa}, \mathrm{x}}
$$

while the apparent vertical angle due to lateral specific force is given by

$$
\varphi_{f}=\operatorname{atan}\left(\frac{\mathrm{f}_{\mathrm{aa}, \mathrm{y}}}{\mathrm{f}_{\mathrm{aa}, \mathrm{z}}}\right)
$$




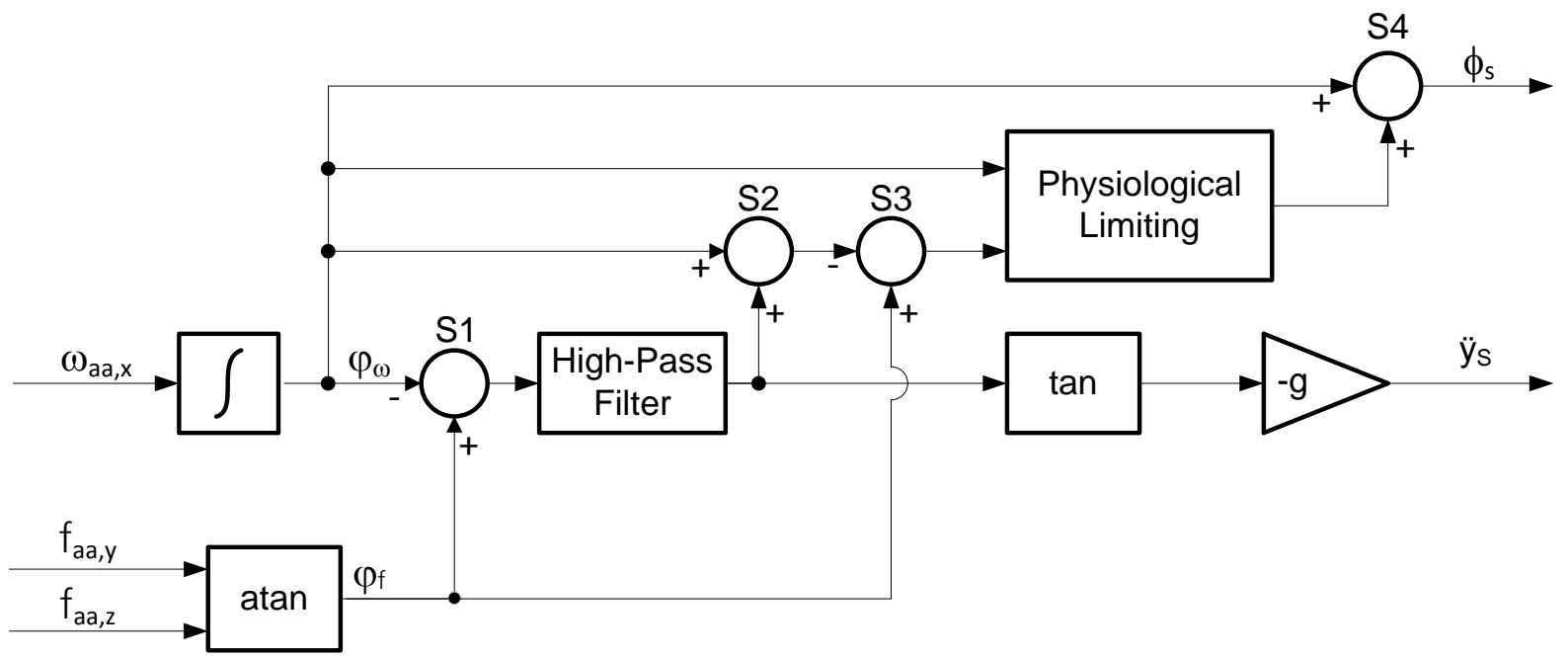

Figure 6. Lateral Channel of an Apparent Vertical Filter according to [10]

For an idealized coordinated turn the apparent vertical angle due to a roll rate matches the roll attitude of the aircraft, while the same angle due to specific force is zero. For this case the AVF processing a lateral maneuver input signal is shown below with all signals greyed-out which are negligible.

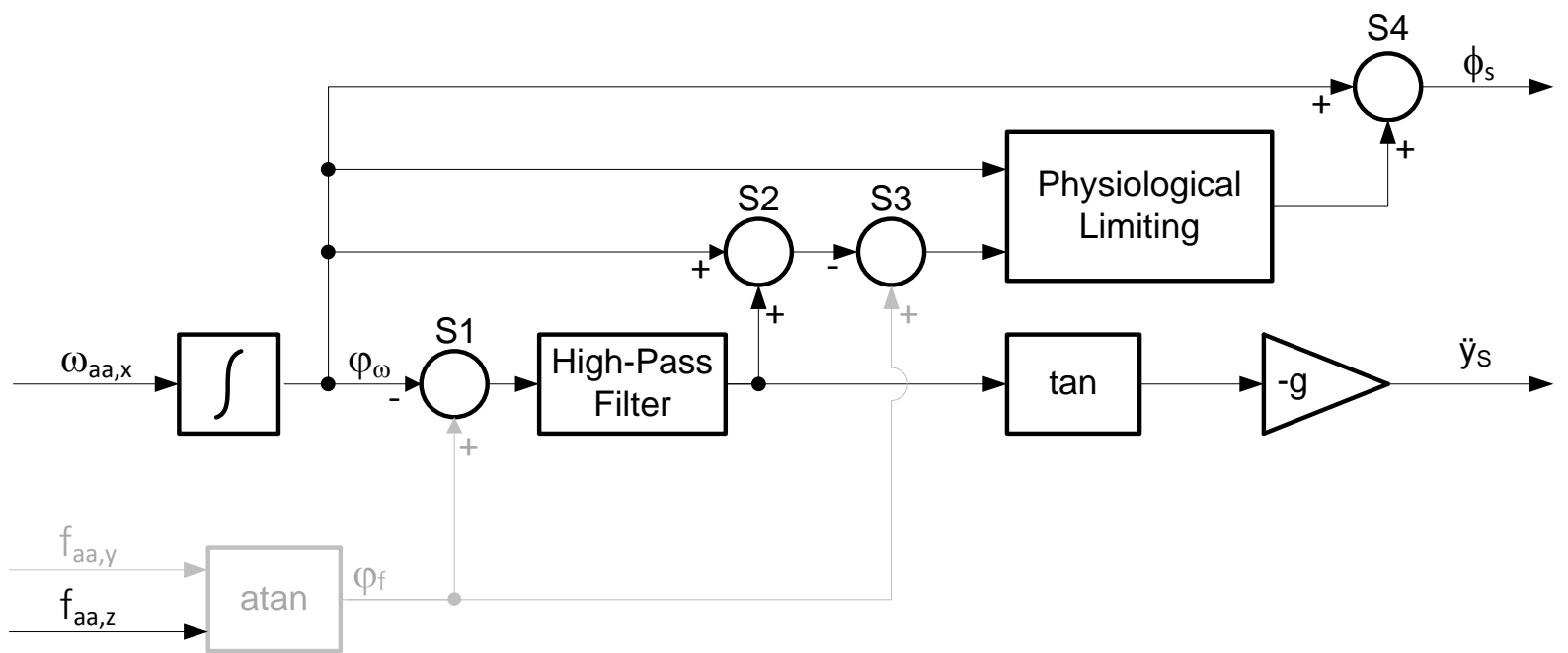

Figure 7. Lateral Channel of an Apparent Vertical Filter processing an idealized coordinated turn

Like other filters the AVF provides a translational and a rotational output signal due to a lateral maneuver input signal. For a coordinated turn the translational response is a high-pass filtered signal of the aircraft attitude. After the 3rd-order high-pass filter the roll angle is transferred back to a lateral acceleration signal that drives the motion platform. The rotational response signal is the sum S4 of the aircraft roll attitude and a low-pass filter signal that, again, leaves a high-pass filtered signal in the roll output signal. The low-pass filtered signal results from the sum S2 of the aircraft roll attitude and the high-pass filtered roll attitude. Because the apparent vertical angle due to specific forces is zero, both differences S1 and S3 do not have any effect on the signal. As a consequence the rotational as well as the translational response signals are filtered the same way and represent the same apparent vertical angle. By doing so the filter response shows a high-pass filtered roll signal and a lateral acceleration that compensates the lateral component of the gravity vector.

One should note that the physiological limiting will only limit the signal in the case of the aircraft and the simulator cabin rotating in different directions. If the simulator cabin rolls back because of the high-pass filter response to a constant roll rate, this might lead to a poorly compensated roll attitude of the simulator and, as a consequence, a false side-force. Therefore for large roll rates the signal needs to be scaled. In addition to that the 
compensatory lateral acceleration is limited because of the limited envelope space available to perform any lateral movement.
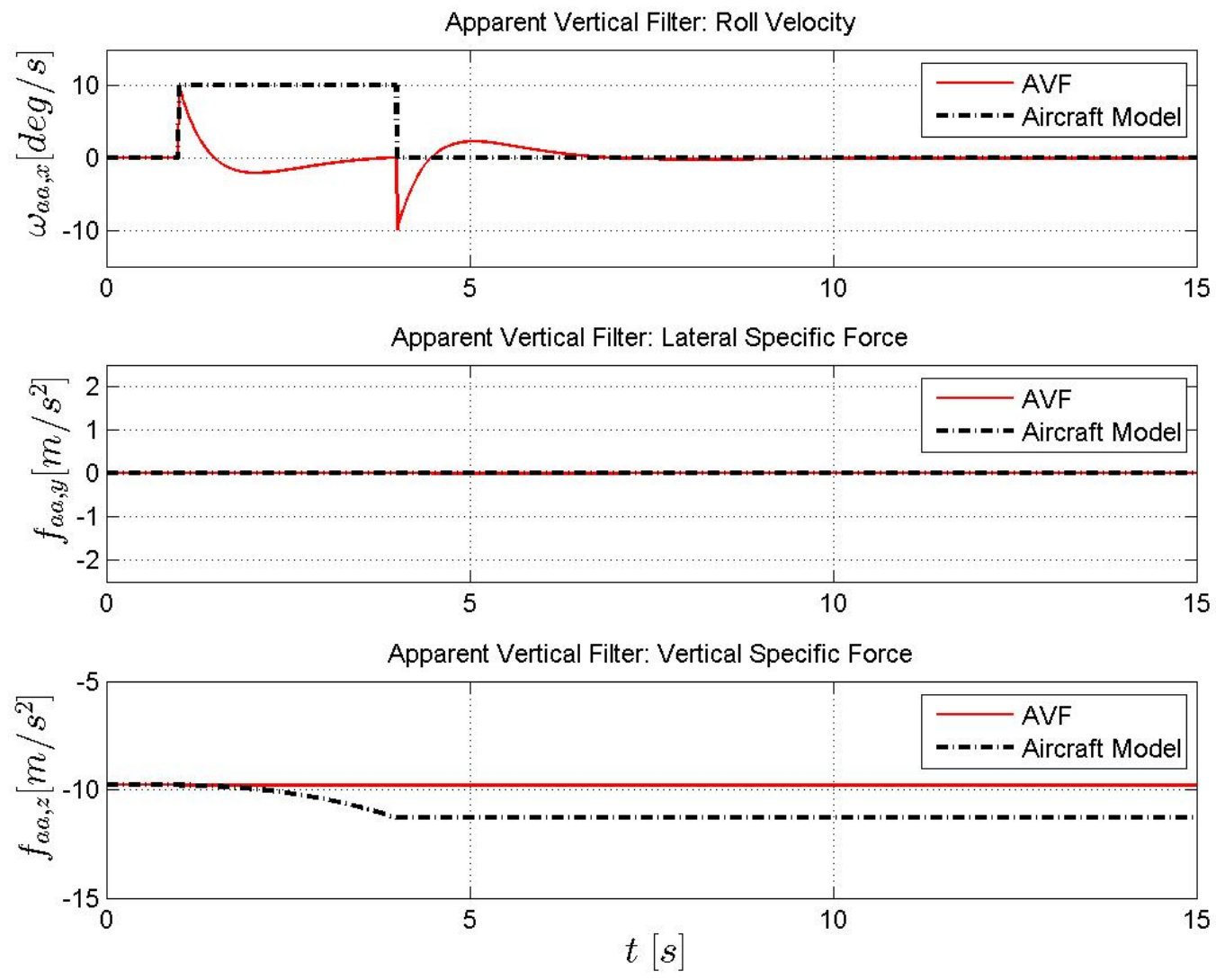

Figure 8. Apparent Vertical Filter reponse to an idealized coordinated turn

Fig. 8 shows the response of the AVF to an idealized coordinated turn input signal. It is easy to see that only very minor side forces occur which should not be noticeable in the simulator while the roll rate at the start of the maneuver will be correctly sensed by the pilot in the simulator. The roll rate as well as the vertical specific force shows only minor differences to those responses found for a CWA.

\section{System Responses to Aircraft Flight Test Data}

After having shown the principle of the AVF in an idealized coordinated turn, the filter response to the outputs of an aircraft simulation model will be demonstrated in this section. The input data used here is flight test data gathered with the DLR Airbus A320 ATRA research aircraft. Again, a turn is used as the reference maneuver for comparing the response of an AVF to that of a CWA. Fig. 9 shows the input signals and the filter response for both filtering methods. The turn was manually controlled. While the first graph shows the specific force magnitude, i.e. the length of the specific force vector, to be represented in the simulator, the second graph presents the apparent vertical angle or the orientation of the force vector. Input data are colored in dashed black, the AVF response is shown in red and that of the CWA is presented in dark blue.

In the first 21 seconds the aircraft remains in level flight with a specific force magnitude close to $\mathrm{g}$ only disturbed by moderate atmospheric turbulences. After approximately 21 seconds the turn starts by rolling the aircraft and the g-force increases. Both the AVF and the CWA are not able to reproduce the vertical load factor for the complete maneuver. Due to the limited motion space available this problem cannot be solved with the hardware design of today's Full Flight Simulators. It is interesting to see that both filter methods show comparable force magnitudes. For a coordinated turn the AVF shows at least similar capabilities as the CWA with respect to this point. 


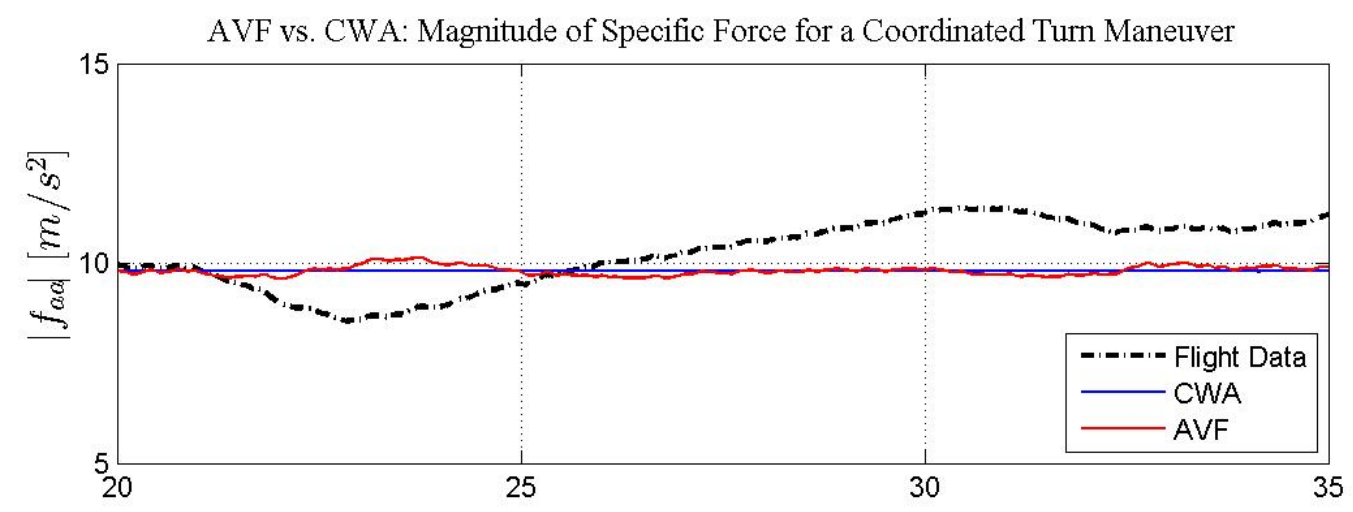

AVF vs. CWA: Apparent Vertical for a Coordinated Turn Maneuver

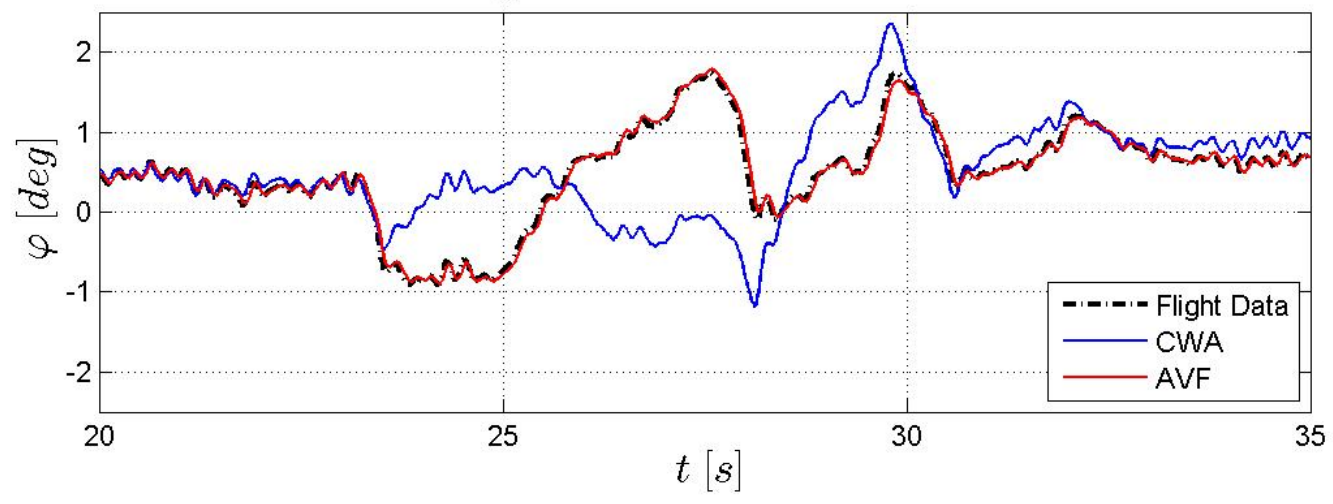

Figure 9. Response of CWA and AVF to an Airbus A320 aircraft turn

Looking at the lower graph the orientation of the force can be analyzed for both methods. At the start of the maneuver the aircraft flew with a small roll attitude of less than $1^{\circ}$. During the whole maneuver, starting from turn initialization to a steady-state coordinated turn, the change of the apparent vertical representing the orientation of the specific force shows little change. During the complete maneuver the apparent vertical in the simulator using the AVF is very close to the input signal from the simulated aircraft. In fact, only small differences due to phase shift effects caused by the signal processing of the filter can be observed.

It should be noted that physiological limits have to be considered in case the simulator cabin rolls differently than the aircraft. If, for example, a lateral specific force occurs due to centrifugal forces during a taxi turn on the ground, it is not possible to reproduce these specific forces in the simulator realistically. A low-frequency side force can, again due to limited motion space, only be represented by a roll attitude of the cabin. Achieving this roll attitude requires a roll rate that the pilot will notice if certain perception thresholds are exceeded. The consequence is a mismatch of visual and motion perception which is seen as a major cause for simulator sickness. For this reason a physiological limiting is needed in order to limit the rotational velocity for those maneuvers at the price of representing the specific forces less accurately.

On the other hand, the system response using the classical washout filter shows some major deviations from the input data. Again, the filter tuning corresponds to a setting of a comparable filter used in an approved Boeing B737 training simulator. It could be argued that these deviations might be the result of an unfavorable tuning. But considering the initial phase of the maneuver it can be observed that the apparent vertical in the simulator cabin not only differs by scale but that the direction of the sensed force is opposite to the input data. This behavior is coherent to the results of the idealized coordinated turn and therefore can be seen as an implicit disadvantage of the CWA. It is not the absolute numbers that justify this statement, it is the opposite direction of the specific force sensed in the cabin during initialization of the turn. 


\section{Conclusions}

The main purpose of the Apparent Vertical Filter is to provide pilots in flight simulators with a better representation of specific forces. Due to the limited envelope space of the motion system, a perfect match of specific forces, especially in terms of force magnitude, is not possible in the simulator. The aim is to provide at least the correct orientation of the force vector. This paper presents the proof-of-concept for the AVF with respect to a coordinated turn maneuver. By defining an idealized test case the specific force input signals are given. The response to those signals is discussed for both a classic washout filter algorithm and the Apparent Vertical Filter. It could be shown that a classical washout filter algorithm is not able to reproduce a coordinated turn without disturbing side forces as long as the roll cue is represented because either the compensating lateral acceleration is high-pass filtered and therefore not optimally compensating the side force due to cabin roll attitude or the simulator cabin is not rotated leading to a good match of specific forces but without any rotational cues felt by the pilot.

The AVF on the other hand is able to roll the simulator cabin and to compensate the g-forces with a lateral acceleration maneuver. Of course this behavior is constrained by the lateral envelope space limiting the roll attitude to be achieved by the simulator cabin. Therefore the roll attitude has to be reduced after the onset of the maneuver back to a neutral position. Nevertheless, at the beginning of the maneuver the pilot feels a roll rate without any side force. Rolling back the cabin will be done below the physiological threshold while any g-force resulting from a cabin roll attitude is compensated for by a corresponding lateral acceleration.

This behavior of the AVF could be demonstrated for both an idealized coordinated turn maneuver and a turn flown in the Airbus A320 ATRA. It could be shown that for both tests the AVF shows an almost perfect match of the apparent vertical orientation comparing input specific forces with the specific forces felt in the simulator.

The example of a turn maneuver demonstrates the major differences in the motion system response of a classical washout filter algorithm and an Apparent Vertical Filter. Due to its inherent design the CWA is not able to represent a roll rate at the initialization of the turn without showing false cues in terms of side forces. The reason for that is the feedback signal of the output roll signal to the translational channel which is, again, high-pass filtered and therefore is not adequately compensating the lateral component of the gravity vector due to the roll attitude of the cabin. This behavior could be shown for an idealized input signal as well as for flight test data. As a consequence, by using the CWA a compromise has to be found between withholding rotational cues from the pilot by not rolling the cabin at all or accepting false lateral acceleration cues by rolling the cabin. In flight training simulators this effect is often suppressed by reducing the gain factor for lateral maneuvers to a value of 0.25 to 0.5 . This can be seen as one important cause why many flight training simulators have problems to represent side forces of lateral maneuvers appropriately.

In contrast to the CWA the Apparent Vertical Filter is able to represent the initialization phase of a turn maneuver with unnoticeable, if any, side forces. Because the specific forces are compared with the aircraft attitudes it considers the maneuver flown. With this information it is possible to decide, for example, whether a lateral gravitational acceleration of the simulator needs to be compensated by a lateral acceleration or not. Hence the sensed apparent vertical angle in the simulator cockpit can match that of an aircraft cockpit as accurately as possible. The magnitude of the specific force, in contrast, has to be limited as with any other filter method because the limited space envelope does not allow a realistic representation.

\section{Outlook}

The behavior of the AVF has been demonstrated with respect to a coordinated turn, one of three lateral maneuvers defined for testing new filter concepts. Other lateral maneuvers are the straight flight with a constant bank angle like the steady heading side-slip and a turn while taxiing on ground. For these maneuvers, as well as for longitudinal and vertical maneuvers, this filter concept will be assessed. Finally, pilot tests and evaluations have to be conducted to prove the overall filter concept. These pilot tests will be carried out in the AVES research simulator located at the DLR site in Braunschweig as well as in training simulators for helicopters and for fixed wing aircraft.

At the moment the AVF filter software corresponds to a prototype. Regarding certain constraints given by the aircraft model it is possible to demonstrate the behavior of the filter for normal flight operation including take-off and landing. To demonstrate the capabilities of the AVF in unsual flight situations like wake encounters or high angle of attack attitudes it is necessary to amend the filter software with envelope protection elements. This functionality is currently under development. 


\section{Acknowledgements}

The authors would like to thank Moog B. V. for their kind cooperation and support of this project. We especially thank Philip van der Borch, Fahmy Abdat and Ashgard Weterdings for their valuable contributions.

\section{References}

${ }^{1}$ Longridge, T., Ray, P., Boothe, E., Bürki-Cohen, J., "Initiative Towards More Affordable Flight Simulators for U.S. Commuter Airline Training,” Proceedings of the Royal Aeronautical Society Conference on Training - Lowering the Cost, Maintaining Fidelity, London, UK, 1996

${ }^{2}$ Bürki-Cohen, J., Sparko, A. L., Jo, Y. J., Go, T. H., "Effects of Visual, Seat, and Platform Motion During Flight Simulator Air Transport Pilot Training and Evaluation,” Proceedings of the $15^{\text {th }}$ International Symposium on Aviation Psychology, Dayton, $\mathrm{OH}, 2009$

${ }^{3}$ Advani, S. K., Hosman, R. J., "Revising Civil Simulator Standards - An Opportunity for Technological Pull,” AIAA Modelling and Simulation Technologies Conference and Exhibit, Keystone, CO, 2006

${ }^{4}$ Hosman, R. J., Advani, S. K., “Are Criteria for Motion Cueing and Time Delays Possible? Part 2,” AIAA Modelling and Simulation Technologies Conference and Exhibit, Boston, MA, 2013

${ }^{5}$ Hodge, S. J., Manso, S., White, M. D., “Challenges in Roll-Sway Motion Cueing Fidelity: A view from academia”, Royal Aeronautical Society Flight Simulation Group Conference, London, UK, 2015

${ }^{6}$ Hodge, S. J., Perfect, P.,Padfield, G. D. White, M. D., "Optimising the roll-sway motion cues available from a short strole hexapod modiot platform,” The Aeronautical Journal, Volume 119, No. 1211, 2015, pp. 23,-44

${ }^{7}$ van Biervliet, F., “A Side Force to Reckon with”, CAT Magazine, Issue 5/2007; pp. 12-13

${ }^{8}$ van Biervliet, F., La Hulpe, Belgium, European Patent Specification for a "Method to Control the Movements of a Flight Simulator and Flight Simulator Implementing such Method,” European Patent No. EP 1946193 B2, filed 12 Oct. 2006

${ }^{9}$ Reid, L. D., Nahon, M. A., "Flight Simulation Motion-Base Drive Algorithms: Part I - Developing and Testing the Equations,” UTIAS Report No. 296, University of Toronto, Toronto, Canada, 1985

${ }^{10}$ Seehof, C., Deutsches Zentrum für Luft- und Raumfahrt e. V., Köln, Germany, German Patent Specification for a “Verfahren und Vorrichtung zur Ansteuerung eines Simulators,” Patent No. DE 102015102 459 B4, filed 03. Nov. 2016 